\title{
Phytoplankton composition and abundance assessment in the Nador lagoon (Mediterranean coast of Morocco)
}

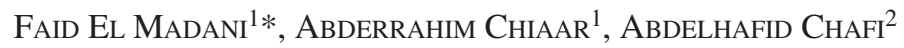 \\ ${ }^{1}$ Institut National de Recherche Halieutique - Centre Régional de Nador, \\ 13 Boulevard Zerktouni, Nador, Morocco \\ ${ }^{2}$ Unité de Formation et de Recherche Techniques de Gestion de l'Environnement, \\ Département de Biologie, Faculté des Sciences, Oujda, Morocco
}

\begin{abstract}
We evaluated phytoplankton abundance, composition and trophic state of the Nador lagoon (Morocco) on the basis of data taken in the period November 2007 to August 2008. Sampling was performed at 11 stations (bottle samples at $0.5 \mathrm{~m}$ depth and horizontal plankton net tows with mesh size of $20 \mu \mathrm{m}$ ). Among seven identified phytoplankton classes, diatoms and dinoflagellates dominated with 133 and 169 species, respectively. Frequent phytoplankton blooms were contributed by one to three species in the lagoon. Abundance and seasonality of phytoplankton characterized the Nador lagoon as a highly eutrophicated environment.
\end{abstract}

Keywords: Mediterranean Sea, Nador lagoon, Phytoplankton, diversity, eutrophication,

\section{Introduction}

Taxonomic composition and size structure of phytoplankton is regulated by eutrophication in coastal lagoons (PÉREZ-RUZAFA et al 2002). Eutrophication can induce massive blooms (coloured water) of phytoplankton species and the reduction in diversity. In our study, both the qualitative and quantitative aspects were considered in order to determine (a) the actual phytoplankton community structure of the Nador lagoon, (b) the spatial distribution and (c) the temporal succession of the dominant species.

\section{Description of the study area}

The Moroccan Nador lagoon is a semi-enclosed coastal ecosystem with a surface area of about $115 \mathrm{~km}^{2}$ (Fig. 1). The lagoon is isolated from the Mediterranean Sea by a $25 \mathrm{~km}$ long sand bar (le Lido), crossed by one channel (Boccana). It is located in a region characterised by a Mediterranean climate with, often, a low and irregular rainfall - (annual mean

* Corresponding author, e-mail: elmadanifaid@hotmail.com

Copyright $^{\circledR} 2011$ by Acta Botanica Croatica, the Faculty of Science, University of Zagreb. All rights reserved. 


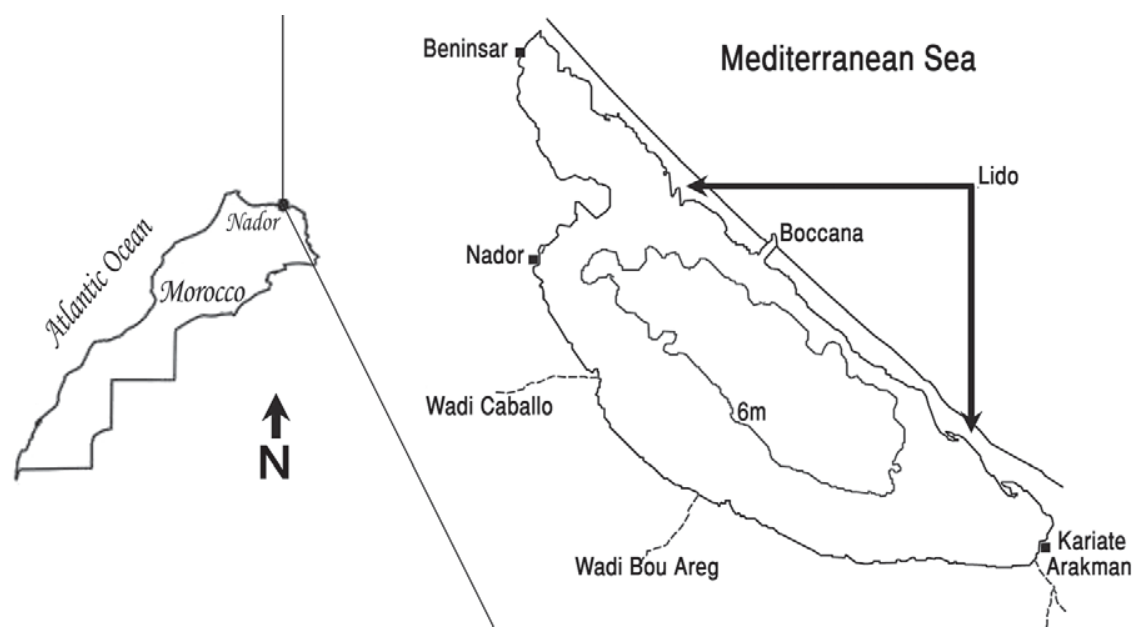

Fig. 1. The bathymetric map of Nador lagoon and its geographic position.

116-430 mm). During the year, there is a distinction between the rainy season from November to March and the dry season from April to October (Fig. 2).

The average depth of the lagoon is $4-7 \mathrm{~m}$ (Fig. 1). The depths increase from edges to the middle part of the lagoon. The salinity range is $32.7-40.2$ in the confined extremity of the lagoon where the mixing of the water column is very low (BENBRAHIM 2009). The water temperature variation closely follows the temperature of the air (LAKHDAR et al. 2005) and generally ranges from $11{ }^{\circ} \mathrm{C}$ in January to $30^{\circ} \mathrm{C}$ in August. Stratification of the water column is weak.

The Nador lagoon is one of the largest costal lagoons on the Mediterranean coast that suffer from a substantial anthropogenic wastewater overload in organic matter, nitrogen and phosphorus in addition to other various kinds of chemicals including heavy metals and

$\begin{array}{lllllllllrlrr}\text { months } & \text { Jan } & \text { Feb } & \text { Mar } & \text { Apr } & \text { May } & \text { Jun } & \text { Jul } & \text { Aug } & \text { Sep } & \text { Oct } & \text { Nov } & \text { Dec } \\ \text { Precipitation }(\mathrm{mm}) & 45.8 & 48.4 & 36.7 & 27.7 & 17.5 & 10.4 & 1.4 & 3 & 9.1 & 22.1 & 32.2 & 32.5 \\ \text { Temperature }\left({ }^{\circ} \mathrm{C}\right) & 12.2 & 13.1 & 14.3 & 15.7 & 18.2 & 21.65 & 24.3 & 25.1 & 23.1 & 19.3 & 16.15 & 13.6\end{array}$

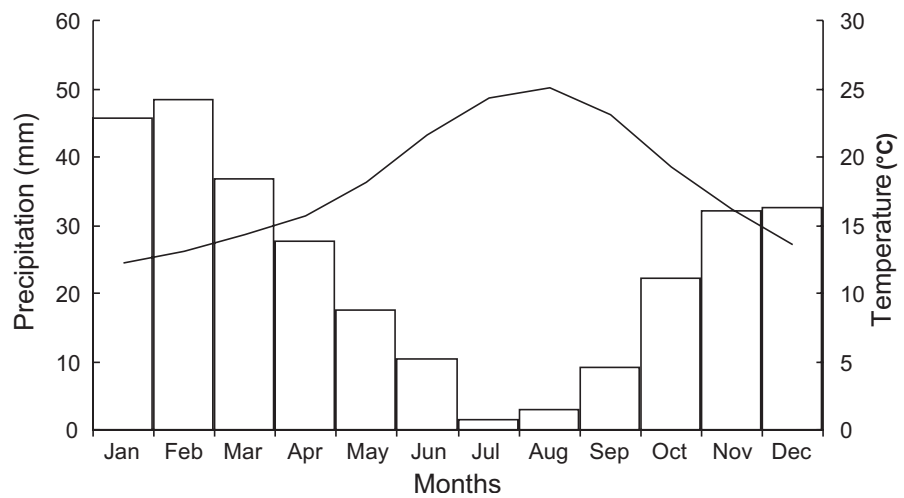

Fig. 2. Monthly mean rainfall and temperature in Nador city, in the period 1977-1996. 
hydrocarbons (BLOUNDI 2005). This organic and inorganic complex may cause dysfunctions in the food web that might lead a total ecosystem imbalance, especially because of the low water exchange rate with the open sea. The turnover time of the water in the lagoon was estimated to be 80 days (HILMI 2005). However, despite the narrowness of the channel, the hydrological balance of this system shows a quasi-permanent predominance by sea water. In 1993 the channel (Boccana) was dredged and widened after a progressive accumulation of the sand that since 1987 had almost completely isolated the lagoon from the open sea; this was done in order to re-establish normal water circulation.

The macrophytes recorded in the lagoon, during the present study, belong to three groups (i) Climax phanerogams Cyamodocea nodosa, and Nanozostera nolti and the Chlorobiontes Caulerpa prolifera, (ii) the opportunist algae: Ulva spp, Enteromorpha spp, Chaetomorpha linum and (iii) the drifting Rhodophyceae species Gracilaria gracilis and Alscidium corlinum. Generally, the invasive macroalgae, Caulerpa prolifera, cover most of the bottom, except the central part that is devoid of macrophytes, restricting the seaweed Cymodocea nodosa to small bands in the shallowest areas surrounding the lagoon.

Diatoms are the dominant planktonic algae in the lagoon (EL MADANI et al 2001).

Several watercourses drain into the lagoon but most of them become functional only during episodic flood periods (MAHJoubi 2003). Some of them, such as Wadi Caballo, Wadi Afelioune and Wadi Akhandouk, became true open wastewater collectors that may contribute to increase the organic matter and various kinds of chemical element input.

\section{Materials and Methods}

Surface phytoplankton sampling was conducted, five times, roughly each two months, at 11 stations along Nador lagoon, (Fig. 3). For qualitative study, samples were collected using a standard plankton net $(20 \mu \mathrm{m}$ mesh size $)$, in a horizontal tow for about 2 minutes at

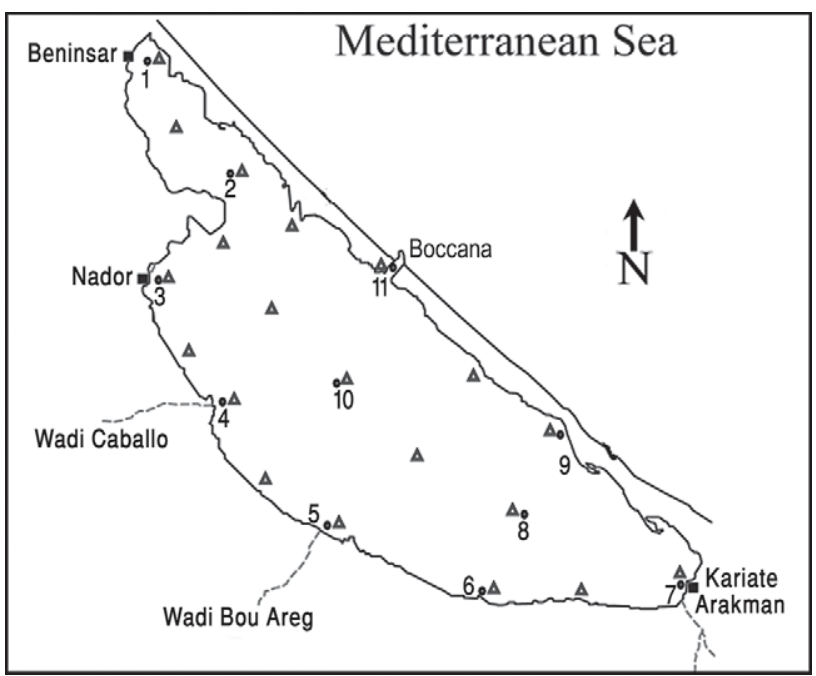

Fig. 3. The sampling plan at stations: phytoplankton $(\bullet)$; temperature and salinity $(\triangle)$. 
each station. For quantitative plankton sampling a bottle was used. Once collected, samples were fixed immediately with neutral formalin. For identification and enumeration of phytoplankton an inverted microscope Leica DM-IRB was used.

Phytoplankton identification was performed according to DODGE (1982), BALECH et al. (1984), Ricard (1987), Balech (1988), Larsen and Moestrup (1989), Delgado and Fortuño (1991), HallegraefF (1991), HallegraefF et al. (1991), Paulmier (1992, 1994), Nezan (1996), Nezan and Piclet (1996), Tomas (1997), Faust et al. (1999), Matsuoka and Fukuyo (2000), HAnsen et al. (2001), SAR et al. (2002), KAshima (2002), KOENING and LIRA (2004).

The salinity and temperature measurements were made in situ (20 stations) with WTW cond-197i model conductivity-salinometer (Fig. 3).

\section{Results}

\section{Water temperature and salinity}

Salinity and temperature increase from January to August (Fig. 4). The salinity minimum is due to the rainfall maximum. If we consider spring the reference season, because of its moderate temperature and low rainfall, the normal salinity in the Nador lagoon is around 36.7 with small variations. The range between minimum and maximum water temperature is about $13{ }^{\circ} \mathrm{C}$, and photoperiod seems to be the main limiting factors for the development of the phytoplankton community.

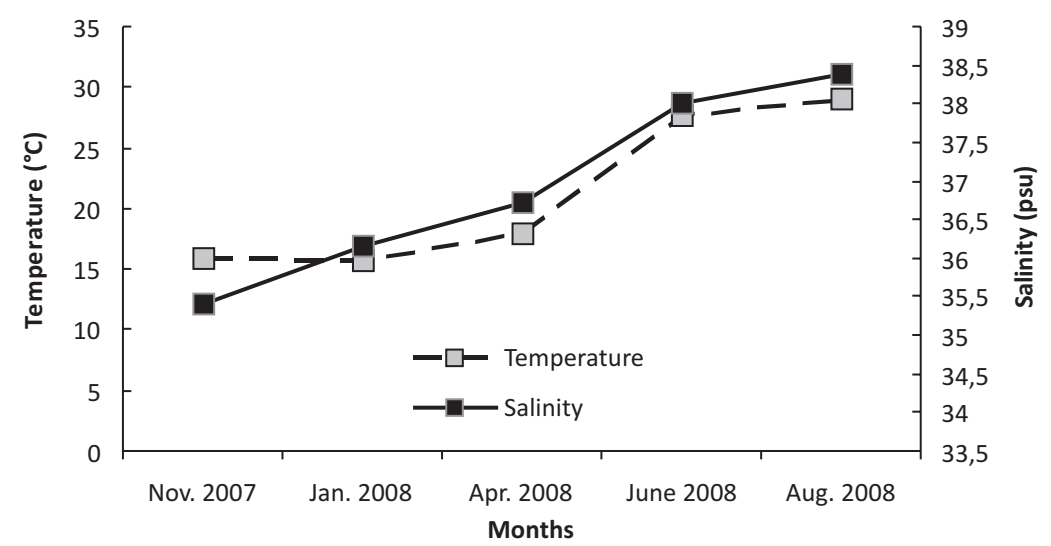

Fig. 4. Temporal variation of the means of the temperature and salinity of the lagoon waters during the study period.

\section{Taxonomic composition}

A total of 311 phytoplankton species belonging to seven groups were identified during the period of study; 133 diatom species, 169 dinoflagellates, 2 cyanophyceae, 2 dyctiochophyceae, 2 euglenophyceae, 1 chlorophyceae, 1 coccolithophorid species and 1 raphydophyceae (Tab. 1). Among phytoplankton species, the most dominant were diatoms, such as: Chaetoceros spp, Pseudonitzschia spp, Nitzschia longissima, Skeletonema sp, Neocera- 
Tab. 1. The spatial distribution of phytoplankton taxa (result from five samplings during 2007 and 2008).

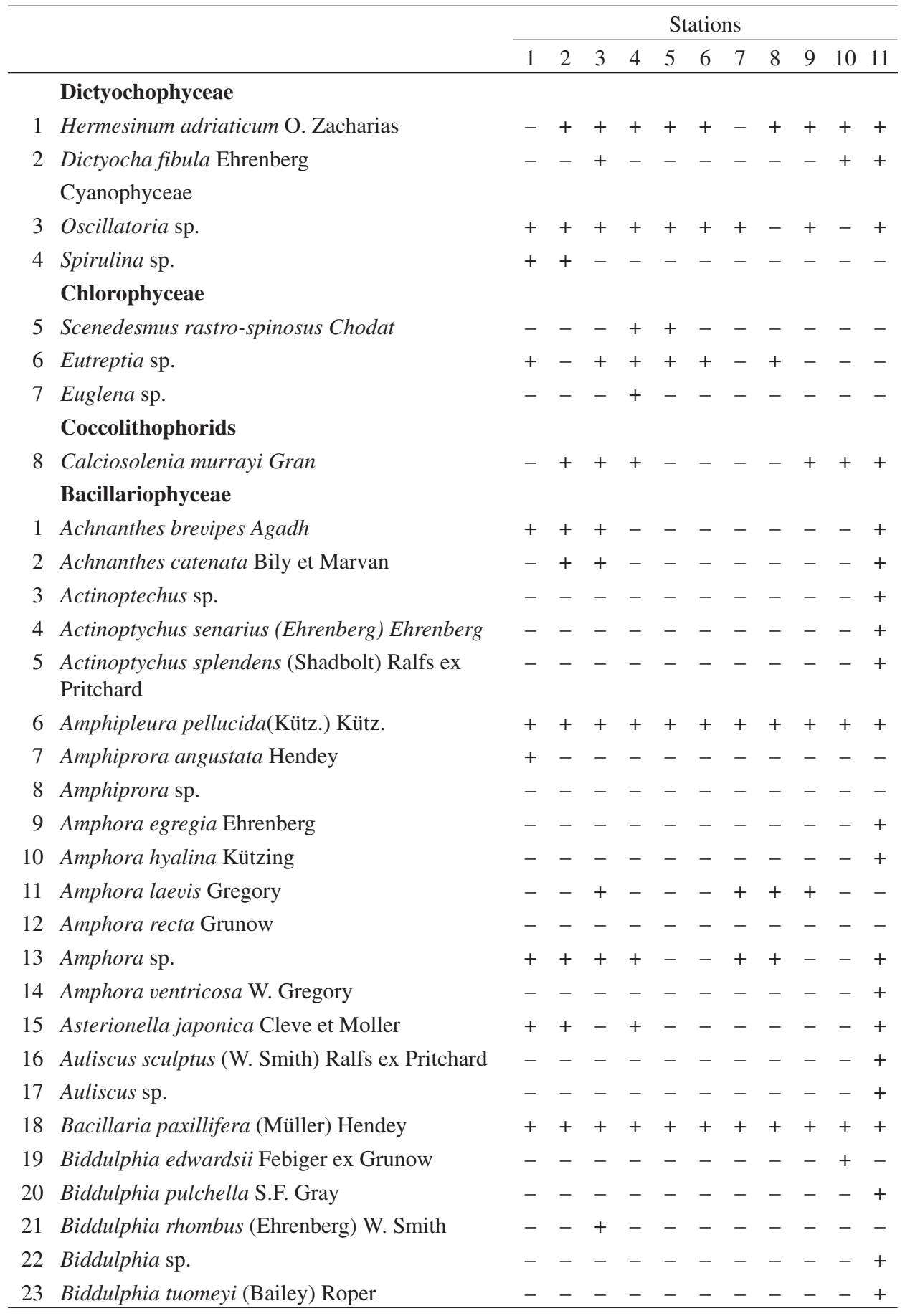


Tab. 1. - continued

\begin{tabular}{|c|c|c|c|c|c|c|c|c|c|c|c|c|}
\hline & & \multicolumn{11}{|c|}{ Stations } \\
\hline & & 1 & 2 & 3 & 4 & 5 & 6 & 7 & 8 & 9 & 10 & 11 \\
\hline 24 & Campylodiscus decorus de Brébisson & - & - & - & - & - & - & - & - & - & - & + \\
\hline 25 & Cerataulina pelagica (Cleve) Hendey & + & + & + & - & + & - & + & + & + & + & + \\
\hline 26 & Chaetoceros affinis var. willei (Gran) Hustedt & - & - & + & - & - & - & - & + & - & - & - \\
\hline 27 & Chaetoceros brevis Shütt & + & - & - & - & - & - & - & - & - & - & - \\
\hline 28 & Chaetoceros curvisetus Cleve & + & + & + & + & - & - & - & - & - & + & - \\
\hline 29 & Chaetoceros danicus Cleve & - & - & + & - & - & + & - & + & - & - & - \\
\hline 30 & Chaetoceros decipiens Cleve & - & + & + & + & + & + & + & + & + & + & + \\
\hline 31 & Chaetoceros didymus Ehrenberg & - & + & - & - & - & - & - & - & - & - & - \\
\hline 32 & Chaetoceros laciniosus Schütt & - & - & - & - & - & - & - & + & - & - & - \\
\hline 33 & Chaetoceros laevis Leuduger-Fortmorel & - & + & - & - & - & - & - & - & - & - & - \\
\hline 34 & Chaetoceros lauderi Ralfs & - & - & - & - & - & + & - & - & - & - & - \\
\hline 35 & Chaetoceros mitra (J.W. Bailey) Cleve & + & + & + & + & - & + & + & + & + & + & + \\
\hline 36 & Chaetoceros peruvianus Brightwell & - & + & + & + & + & - & - & + & + & + & + \\
\hline 37 & Chaetoceros pseudocurvisetus Mangin & - & + & - & - & - & - & - & - & - & - & - \\
\hline 38 & Chaetoceros simplex Ostenfeld & - & - & - & + & - & - & - & - & - & - & - \\
\hline 39 & Chaetoceros spp. & + & + & + & + & + & + & + & + & + & + & + \\
\hline 40 & Chaetoceros teres Cleve & - & + & - & + & - & + & + & - & - & - & + \\
\hline 41 & Chrysanthemodiscus floriatus Mann & - & - & - & - & - & - & - & - & - & - & + \\
\hline 42 & Climaconeis sp. & - & - & - & - & - & - & + & - & - & - & - \\
\hline 43 & Cocconeis scutellum Ehrenberg & - & + & - & - & - & + & - & + & - & - & + \\
\hline 44 & Cocconeis sp. & + & - & + & - & - & - & - & - & + & - & + \\
\hline 45 & Corethron criophilum Castracane & - & + & - & - & - & - & - & - & - & - & - \\
\hline 46 & Coscinodiscus sp. & - & - & + & - & - & - & - & - & - & - & + \\
\hline 47 & Dactyliosolen fragilissimus (Bergon) G. R. Hasle & - & - & - & + & - & - & - & - & - & + & - \\
\hline 48 & Diatoma mesodon (Ehrenberg) Kützing & - & - & - & - & - & - & - & - & - & - & + \\
\hline 49 & Diploneis chersonensis (Grunow) Cleve & - & - & - & - & - & - & - & - & - & - & + \\
\hline 50 & Diploneis sp. & - & - & - & - & - & - & - & - & + & - & - \\
\hline 51 & Ditylum brightwellii (T. West) Grunow & + & - & + & - & - & - & - & - & - & + & - \\
\hline 52 & Entomoneis alata (Ehrenberg) Ehrenberg & + & - & + & - & + & + & + & - & + & - & - \\
\hline 53 & Entomoneis sp. & + & + & - & - & - & - & - & - & - & - & - \\
\hline 54 & Epithemia turgida (Ehrenberg) Kütz & - & - & - & - & - & - & - & - & - & - & + \\
\hline 55 & Goniothecium odontella Ehrenberg & - & - & - & - & - & - & - & - & - & - & + \\
\hline 56 & Grammatophora angulosa Ehrenberg & - & - & - & - & - & - & - & - & - & - & + \\
\hline 57 & Grammatophora marina (Lyngbye) Kützing & - & + & + & - & - & - & - & - & - & - & - \\
\hline 58 & Guinardia delicatula (Cleve) Hasle & - & - & - & - & - & + & + & - & - & - & + \\
\hline 59 & Guinardia flaccida (Castracane) H. Peragallo & + & - & - & - & - & - & - & - & - & - & + \\
\hline 60 & Guinardia striata (Stoltherfoth) Hasle & - & + & + & - & + & + & + & + & + & + & + \\
\hline
\end{tabular}


Tab. 1. - continued

\begin{tabular}{|c|c|c|c|c|c|c|c|c|c|c|c|c|}
\hline & & \multicolumn{11}{|c|}{ Stations } \\
\hline & & 1 & 2 & 3 & 4 & 5 & 6 & 7 & 8 & 9 & 10 & 11 \\
\hline 61 & $\begin{array}{l}\text { Gyrosigma fasciola (Ehrenberg) J.W.Griffith } \\
\text { et Henfrey }\end{array}$ & + & + & - & + & + & - & + & - & - & + & - \\
\hline 62 & Gyrosigma scalproide (Rabenhorst) Cleve & - & + & - & + & - & - & - & - & + & - & + \\
\hline 63 & Gyrosigma sp. & - & - & - & + & - & + & - & - & - & - & - \\
\hline 64 & Gyrosigma wansbeckii (Donkin) Cleve & - & - & - & - & + & - & - & - & - & - & - \\
\hline 65 & Hantzschia amphioxus (Ehrenberg) Grunow & - & - & - & - & - & - & - & - & - & - & + \\
\hline 66 & Haslea wawrikae (Husedt) Simonsen & - & + & + & + & - & + & - & - & - & - & + \\
\hline 67 & Hemialus hauckii Grunow ex Van Heurck & + & + & + & + & + & + & + & - & + & + & + \\
\hline 68 & Hemiaulus sinensis Greville & - & - & - & - & + & - & - & - & - & - & - \\
\hline 69 & Lauderia annulata Cleve & - & - & - & - & - & - & - & - & - & - & + \\
\hline 70 & Lauderia sp. & - & - & - & - & - & - & - & - & + & - & - \\
\hline 71 & Leptocylindrus danicus Cleve & - & + & + & + & - & + & - & + & + & + & + \\
\hline 72 & Leptocylindrus minimus Gran & - & + & + & + & + & + & + & + & + & + & + \\
\hline 73 & Licmophora flabellata (Greville) Agardh & + & + & + & + & + & + & + & + & + & + & + \\
\hline 74 & Licmophora gracilis (Ehrenberg) Grunow & + & + & + & + & + & + & - & - & - & + & + \\
\hline 75 & Licmophora sp. & + & + & - & - & - & - & - & - & - & + & + \\
\hline 76 & Lioloma pacificum (Cupp) Hasle & - & - & - & + & - & + & - & - & - & + & - \\
\hline 77 & Lioloma sp. & - & + & - & - & - & - & - & - & - & - & - \\
\hline 78 & Melosira nummuloides C.A. Agardh & - & + & - & - & - & - & - & - & - & - & - \\
\hline 79 & Melosira sp. & - & - & - & - & - & - & - & - & - & + & + \\
\hline 80 & $\begin{array}{l}\text { Meuniera membranacea (Cleve) P.C. Silva in } \\
\text { Hasle et Syvertsen }\end{array}$ & + & - & - & - & - & - & - & - & - & - & + \\
\hline 81 & Navicula cf. carinifera Grun. Grunow in Schmidt & - & + & - & - & - & - & - & - & - & - & - \\
\hline 82 & Navicula forcipata Greville & - & - & - & - & - & - & - & - & - & - & + \\
\hline 83 & Navicula hasta Pantocsek & - & - & - & - & - & - & - & - & - & - & + \\
\hline 84 & Navicula hennedyi W.Smith & - & - & - & - & - & - & - & - & - & - & + \\
\hline 85 & Navicula humerosa Brébisson ex W. Smith & - & - & - & - & - & - & - & - & - & - & + \\
\hline 86 & Navicula lanceolata (C. Agardh) Kützing & - & - & - & - & - & - & - & - & - & - & + \\
\hline 87 & Navicula menaiana Hendey & - & - & - & - & - & - & - & - & - & - & + \\
\hline 88 & $\begin{array}{l}\text { Navicula smithii Navicula smithii (Agardh) Van } \\
\text { Heurck }\end{array}$ & - & - & - & - & - & - & - & - & - & - & + \\
\hline 89 & Navicula spp. & - & + & + & - & + & + & - & - & + & - & + \\
\hline 90 & Navicula tuscula (Ehrenberg) Grunow & - & + & - & - & - & - & - & - & - & - & - \\
\hline 91 & $\begin{array}{l}\text { Cylindrotheca closterium (Ehrenberg) Reimann } \\
\text { et J.C.Lewin Kingston }\end{array}$ & - & + & + & + & + & + & + & - & + & + & + \\
\hline 92 & $\begin{array}{l}\text { Nitzschia levidensis (W. Smith) Grunow in Cleve } \\
\text { et Grunow }\end{array}$ & - & - & - & - & - & - & - & - & - & - & + \\
\hline 93 & Nitzschia longissima (Brébisson) Ralfs & + & + & + & + & + & + & + & + & + & + & + \\
\hline
\end{tabular}


Tab. 1. - continued

\begin{tabular}{|c|c|c|c|c|c|c|c|c|c|c|c|}
\hline & \multicolumn{11}{|c|}{ Stations } \\
\hline & 1 & 2 & 3 & 4 & 5 & 6 & 7 & 8 & 9 & 10 & 11 \\
\hline 94 Nitzschia panduriformis W. Gregory & - & - & - & - & - & - & - & - & - & - & + \\
\hline 95 Nitzschia sigma (Kützing) W. Smith & - & + & - & - & - & + & - & - & - & - & + \\
\hline 96 Nitzschia sp. & - & + & + & + & + & + & + & + & + & + & + \\
\hline 97 Nitzschia ventricosa Kitton & + & + & - & - & + & + & + & + & + & + & + \\
\hline 98 Oestrupia musca (Gregory) Hustedt & - & - & - & - & - & - & - & - & - & - & + \\
\hline 99 Plagiotropis lepidoptera (Greg.) Reimer Navarro & - & - & - & - & - & - & + & - & - & - & - \\
\hline 100 Planctoniella sol (Wallich) Schutt & - & - & - & - & - & - & - & - & - & - & + \\
\hline 101 Pleurosigma elangatum W. Smith & + & + & + & - & + & + & + & + & + & + & + \\
\hline 102 Pleurosigma itium Ricard & - & + & + & + & + & + & + & - & + & + & + \\
\hline 103 Pleurosigma spp. & + & - & + & + & - & - & - & - & - & - & + \\
\hline 104 Podocystis adriatica (Kützing) Ralfs & - & + & - & - & - & - & - & - & - & - & + \\
\hline 105 Proboscia alata (Brightwell) Sundström & - & - & - & - & - & - & - & - & - & - & + \\
\hline 106 Pseudoguinardia recta von Stosch & - & - & - & - & - & - & - & - & - & + & + \\
\hline 107 Pseudo-nitzschia spp. & + & + & + & + & + & + & + & + & + & + & + \\
\hline 108 Rhizosolenia alata Brightwell & - & - & - & - & - & - & - & - & - & - & + \\
\hline 109 Rgizosolena setigera Brightwell & - & + & - & + & + & - & - & + & - & + & - \\
\hline 110 Rhizosolenea sp. & - & - & - & + & - & - & - & - & - & - & - \\
\hline $\begin{array}{l}111 \text { Rhizosolenia alata forma indica } \\
\text { (H. Peragallo) Gran }\end{array}$ & - & - & - & - & - & - & - & - & + & - & + \\
\hline 112 Rhizosolenia setigera Brightwell & + & + & + & + & + & + & - & + & + & + & + \\
\hline 113 Rhizosolenia sp. & - & - & - & - & - & - & - & - & - & - & + \\
\hline 114 Rhizosolenia styliformis Brightwell & - & - & - & - & + & + & - & - & - & + & + \\
\hline 115 Rhoicosigma sp. & - & - & - & - & - & - & - & - & - & - & + \\
\hline 116 Skeletonema sp. & - & + & + & + & + & + & + & + & + & + & + \\
\hline 117 Stauroneis amphioxys Gregory & - & - & - & - & - & - & - & - & - & - & + \\
\hline $\begin{array}{l}118 \text { Stenopterobia intermedia (Lewis) Brébisson } \\
\text { ex Van Heurck }\end{array}$ & - & - & - & - & - & - & - & - & - & - & + \\
\hline 119 Helicotheca tamesis Ricard & - & - & - & + & - & - & - & - & - & - & - \\
\hline 120 Striatella unipunctata (Lyngbye) C. Agardh & + & + & + & + & - & - & + & - & - & - & + \\
\hline 121 Surirella amoricana $\mathrm{H}$. Peragallo & - & - & - & - & - & - & - & - & - & - & + \\
\hline 122 Surirella fastuosa Ehrenberg & - & - & - & + & + & + & - & - & - & + & + \\
\hline 123 Surirella gemma (Ehrenberg) Kützing & - & + & + & + & - & + & + & - & - & - & - \\
\hline 124 Surirella sp. & - & - & - & - & - & - & - & - & - & - & + \\
\hline 125 Synedra sp. & + & - & - & - & - & - & - & - & - & - & - \\
\hline $\begin{array}{l}126 \text { Thalassionema nitzschioides (Grunow) } \\
\text { Mereschkowsky }\end{array}$ & + & + & + & + & + & + & + & + & + & + & + \\
\hline 127 Thalassiosira hyalina (Grunow) Gran & - & - & - & - & - & - & - & - & - & - & + \\
\hline 128 Thalassiosira sp. & + & + & - & - & + & - & - & - & - & + & + \\
\hline
\end{tabular}


Tab. 1. - continued

\begin{tabular}{|c|c|c|c|c|c|c|c|c|c|c|c|c|}
\hline & & \multicolumn{11}{|c|}{ Stations } \\
\hline & & 1 & 2 & 3 & 4 & 5 & 6 & 7 & 8 & 9 & 10 & 11 \\
\hline 129 & Thalassiothrix froenfeldeii Grunow & + & + & + & - & + & - & + & - & + & - & + \\
\hline 130 & Thalassiothrix spp. & + & + & + & + & + & + & + & + & + & + & + \\
\hline 131 & Toxonidea sp. & - & - & - & - & - & - & - & - & - & - & + \\
\hline 132 & Triceratium alternans J. W. Bailey & - & - & - & - & - & - & - & - & + & - & + \\
\hline 133 & $\begin{array}{l}\text { Trichotoxon reinboldii (Van Heurck) Reid et } \\
\text { Round }\end{array}$ & - & + & - & - & - & - & - & - & - & + & - \\
\hline & Dinoflagellates & & & & & & & & & & & \\
\hline 1 & Achradina pulchra Lohmann & + & + & - & - & - & + & + & - & - & - & - \\
\hline 2 & $\begin{array}{l}\text { Alexandrium catenella (Whedon et Kofoid) } \\
\text { Balech }\end{array}$ & - & - & + & + & - & - & - & - & - & - & + \\
\hline 3 & Alexandrium margalefi Balech & - & - & - & - & - & - & - & - & - & + & + \\
\hline 4 & Alexandrium minutum Halim & + & + & + & + & + & + & + & + & + & + & + \\
\hline 5 & $\begin{array}{l}\text { Alexandrium pseudogonyaulax (Biecheler) } \\
\text { Horiguchi ex Yuki et Fukuyo }\end{array}$ & - & + & + & - & - & + & - & + & + & + & + \\
\hline 6 & Alexandrium sp. & - & - & - & - & - & - & - & - & + & - & + \\
\hline 7 & Alexandrium tamarense (Lebour) E.Balech & - & - & - & - & - & - & + & - & - & - & - \\
\hline 8 & Amphidinium sp. & - & - & - & + & + & - & - & - & - & - & - \\
\hline 9 & Amphidoma caudata Halldal & + & + & + & + & + & + & + & + & + & + & + \\
\hline 10 & Amylax sp. & - & - & - & + & - & - & - & - & - & - & - \\
\hline 11 & Archaeperidinium sp. & - & - & - & - & - & - & - & - & - & - & + \\
\hline 12 & Coolia monotis Meunier & + & + & + & - & - & - & + & + & - & + & + \\
\hline 13 & Cyst Alexandrium minutum & - & - & - & + & + & + & - & + & + & + & - \\
\hline 14 & Cyst of dinoflagellates & + & + & + & - & - & + & + & + & + & + & + \\
\hline 15 & Dinophysis caudata Saville-Kent & - & + & - & - & - & - & + & + & + & + & + \\
\hline 16 & $\begin{array}{l}\text { Dinophysis contracta (Kofoid et Skogsberg) } \\
\text { Balech }\end{array}$ & - & - & - & - & - & - & - & - & - & - & + \\
\hline 17 & Dinophysis diegensis Kofoid & - & - & - & - & - & - & - & - & - & - & + \\
\hline 18 & Dinophysis exigua Kofoid and Skogsberg & - & - & + & - & - & - & - & - & + & + & + \\
\hline 19 & Dinophysis rapa (Stein) Balech & - & - & - & - & - & - & - & - & - & - & + \\
\hline 20 & Dinophysis rotundata Claparède et Lachmann & - & - & - & - & - & - & - & - & - & - & + \\
\hline 21 & Dinophysis sacculus Stein & + & + & + & + & + & + & + & + & + & + & + \\
\hline 22 & Dinophysis sp. & - & - & - & - & - & - & - & - & - & - & + \\
\hline 23 & Diplopelta asymetrica (Mangin) Lindermann & - & - & - & - & + & - & - & + & - & - & - \\
\hline 24 & Diplopelta steinii (T. H. Abé) E. Balech & - & - & - & - & - & - & - & - & - & - & + \\
\hline 25 & Diplopeltopsis minor Pavillard & - & + & - & - & - & - & - & - & - & - & + \\
\hline 26 & Diplopeltopsis sp. & - & + & - & - & - & - & - & - & - & - & - \\
\hline 27 & Diplopsalis sp. & + & + & + & - & + & + & + & + & + & + & + \\
\hline 28 & $\begin{array}{l}\text { Diplopsalopsis bomba (Stein ex Jörgensen) } \\
\text { J. D. Dodge et S. Toriumi }\end{array}$ & - & - & - & - & - & - & - & - & + & - & - \\
\hline
\end{tabular}


Tab. 1. - continued

\begin{tabular}{|c|c|c|c|c|c|c|c|c|c|c|c|c|}
\hline & & \multicolumn{11}{|c|}{ Stations } \\
\hline & & 1 & 2 & 3 & 4 & 5 & 6 & 7 & 8 & 9 & 10 & 11 \\
\hline 29 & Diplopsalopsis sp. & - & - & - & - & - & - & - & - & - & + & - \\
\hline 30 & Ensciculifera sp. & + & + & + & + & - & + & + & + & + & - & + \\
\hline 31 & Ensiculifera angulata E. Balech & - & - & - & - & - & - & - & - & - & + & - \\
\hline 32 & Ensiculifera angulata $\mathrm{E}$. Balech & - & - & - & - & - & - & - & - & - & - & + \\
\hline 33 & Gambierdiscus toxicus Adachi and Fukuyo & + & - & - & - & - & - & - & - & - & - & - \\
\hline 34 & Goniodoma polyedricum (Pouchet) Jörgensen & - & - & + & - & - & - & - & - & - & + & + \\
\hline 35 & Gonyaulax dicantha (Meunier) Schiller & - & - & + & + & + & + & + & + & + & - & + \\
\hline 36 & Gonyaulax digitale (Pouchet) Kofoid & + & - & - & + & - & + & + & + & - & + & + \\
\hline 37 & Gonyaulax grindleyi Reinecke & - & - & - & - & - & - & - & + & - & - & + \\
\hline 38 & Gonyaulax polygramma Stein & - & - & - & - & - & + & - & - & + & + & + \\
\hline 39 & Gonyaulax sousae Balech & + & + & + & + & + & + & + & + & + & + & + \\
\hline 40 & $\begin{array}{l}\text { Gonyaulax spinifera (Claparède et Lachmann) } \\
\text { Diesing }\end{array}$ & + & + & + & + & + & + & + & + & + & + & + \\
\hline 41 & Gonyaulax striata Mangin & - & - & - & - & - & - & - & - & - & - & + \\
\hline 42 & Gonyaulax turbynei Murray et Whitting & - & - & - & - & - & - & - & - & - & + & + \\
\hline 43 & Gonyaulax unicornis Lebour & + & + & - & + & + & - & + & + & + & - & + \\
\hline 44 & Gonyaulax veriore Sournia & - & - & - & - & - & - & - & + & + & - & - \\
\hline 45 & Gotoius mutsuensis Abé & - & - & - & - & - & - & - & - & - & + & - \\
\hline 46 & Gymnodinium catenatum Graham & - & + & - & + & - & - & - & - & - & - & - \\
\hline 47 & Gymnodinium sanguineum $\mathrm{K}$. Hira & + & + & + & + & + & + & + & + & + & + & + \\
\hline 48 & Gymnodinium sp. & - & + & - & - & - & - & + & + & - & + & + \\
\hline 49 & Gyrodinium sp. & - & - & + & + & - & - & - & + & - & - & + \\
\hline 50 & $\begin{array}{l}\text { Gyrodinium spirale (Bergh) } \\
\text { Kofoid et Swezy }\end{array}$ & - & + & + & + & - & + & + & + & + & + & - \\
\hline 51 & Heterocapsa circularisquama Horiguchi & - & - & - & - & - & - & - & - & - & + & - \\
\hline 52 & $\begin{array}{l}\text { Heterocapsa niei (Loeblich) Morrill et } \\
\text { Loeblich III }\end{array}$ & - & - & - & + & - & - & - & + & + & + & - \\
\hline 53 & Heterocapsa rotundata (Lohmann) G. Hansen & - & - & - & - & - & - & - & + & + & + & - \\
\hline 54 & Heterocapsa triquetra (Ehrenberg) F. Stein & - & - & + & - & - & - & - & - & - & - & - \\
\hline 55 & Katodinium sp. & - & + & - & - & - & + & + & - & - & + & + \\
\hline 56 & Lingulodinium polyedrum (Stein) Dodge & + & + & + & + & + & + & + & + & + & + & + \\
\hline 57 & $\begin{array}{l}\text { Neoceratium azoricum (Cleve) F. Gómez, } \\
\text { D. Moreira et P. López-García }\end{array}$ & - & - & - & - & - & - & - & - & - & - & + \\
\hline 58 & $\begin{array}{l}\text { Neoceratium candelabrum (Ehrenberg) } \\
\text { F. Gómez, D. Moreira et P. López-García }\end{array}$ & - & - & - & - & - & - & - & - & - & - & + \\
\hline 59 & $\begin{array}{l}\text { Neoceratium contrarium (Gourret) } \\
\text { F. Gómez, D. Moreira et P. López-García }\end{array}$ & - & + & - & - & - & - & - & - & - & + & + \\
\hline 60 & $\begin{array}{l}\text { Neoceratium declinatum f. declinatum (Sournia) } \\
\text { F. Gómez, D. Moreira et P. López-García }\end{array}$ & - & - & - & - & - & - & - & - & - & + & - \\
\hline
\end{tabular}


Tab. 1. - continued

\begin{tabular}{|c|c|c|c|c|c|c|c|c|c|c|c|c|}
\hline & & \multicolumn{11}{|c|}{ Stations } \\
\hline & & 1 & 2 & 3 & 4 & 5 & 6 & 7 & 8 & 9 & 10 & 11 \\
\hline 61 & $\begin{array}{l}\text { Neoceratium declinatum f. majus (Jörgensen) } \\
\text { F. Gómez, D. Moreira et P. López-García }\end{array}$ & - & - & - & - & - & - & - & - & - & + & - \\
\hline 62 & $\begin{array}{l}\text { Neoceratium deflexum (Kofoid) } \\
\text { F. Gómez, D. Moreira et P. López-García }\end{array}$ & - & - & - & - & - & - & - & - & - & - & + \\
\hline 63 & $\begin{array}{l}\text { Neoceratium extensum (Gourret) } \\
\text { F. Gómez, D. Moreira et P. López-García }\end{array}$ & - & - & - & - & - & - & - & - & - & - & + \\
\hline 64 & $\begin{array}{l}\text { Neoceratium furca (Ehrenberg) } \\
\text { F. Gómez, D. Moreira et P. López-García }\end{array}$ & + & + & + & + & + & + & + & + & + & + & + \\
\hline 65 & $\begin{array}{l}\text { Neoceratium fusus (Ehrenberg) } \\
\text { F. Gómez, D. Moreira et P. López-García }\end{array}$ & - & + & + & + & - & - & - & - & + & + & + \\
\hline 66 & $\begin{array}{l}\text { Neoceratium karstenii (Pavillard) } \\
\text { F. Gómez, D. Moreira et P. López-García }\end{array}$ & - & - & - & - & - & - & - & - & - & - & + \\
\hline 67 & $\begin{array}{l}\text { Neoceratium lineatum (Ehrenberg) } \\
\text { F. Gómez, D. Moreira et P. López-García }\end{array}$ & - & - & - & - & - & - & - & - & - & - & + \\
\hline 68 & $\begin{array}{l}\text { Neoceratium longipes (Bailey) } \\
\text { F. Gómez, D. Moreira et P. López-García }\end{array}$ & - & - & - & - & - & - & - & - & - & - & + \\
\hline 69 & $\begin{array}{l}\text { Neoceratium macroceros (Ehrenberg) } \\
\text { F. Gómez, D. Moreira et P. López-García }\end{array}$ & - & + & - & - & - & - & - & - & - & + & - \\
\hline 70 & $\begin{array}{l}\text { Neoceratium massiliens (Gourret) } \\
\text { F. Gómez, D. Moreira et P. López-García }\end{array}$ & - & - & - & - & - & - & - & - & - & + & + \\
\hline 71 & $\begin{array}{l}\text { Neoceratium massiliens armatum (Karsten) } \\
\text { F. Gómez, D. Moreira et P. López-García }\end{array}$ & - & - & - & - & - & - & - & - & - & - & + \\
\hline 72 & $\begin{array}{l}\text { Neoceratium pentagonum var. tenerum } \\
\text { (Jörgensen) F. Gómez, D. Moreira et } \\
\text { P. López-García }\end{array}$ & - & - & - & - & - & - & - & - & - & - & + \\
\hline 73 & $\begin{array}{l}\text { Neoceratium teres (Kofoid) } \\
\text { F. Gómez, D. Moreira et P. López-García }\end{array}$ & - & - & - & - & - & - & - & - & + & - & - \\
\hline 74 & $\begin{array}{l}\text { Neoceratium trichoceros (Ehrenberg) } \\
\text { F. Gómez, D. Moreira et P. López-García }\end{array}$ & - & + & + & - & - & - & - & + & - & + & + \\
\hline 75 & $\begin{array}{l}\text { Neoceratium tripos (O. F. Müller) } \\
\text { F. Gómez, D. Moreira et P. López-García }\end{array}$ & - & - & + & - & - & - & - & - & + & - & - \\
\hline 76 & $\begin{array}{l}\text { Neoceratium tripos f. tripodoides (Jörgensen) } \\
\text { F. Gómez, D. Moreira et P. López-García }\end{array}$ & - & - & - & - & - & - & - & - & - & - & + \\
\hline 77 & $\begin{array}{l}\text { Noctiluca scintillans (Macartney) Kofoid et } \\
\text { Swezy }\end{array}$ & - & + & + & + & + & + & - & + & + & + & + \\
\hline 78 & $\begin{array}{l}\text { Oblea baculifera Balech ex Loeblich Jr. et } \\
\text { Loeblich III }\end{array}$ & + & - & + & + & + & + & + & + & + & + & + \\
\hline 79 & Ornithocercus magnificus Stein & - & - & - & - & - & - & - & - & - & - & + \\
\hline 80 & Ostreopsis ovata Fukuyo & - & - & + & - & - & - & - & - & - & - & + \\
\hline 81 & Palaeophalacroma unicinctum Schiller & - & - & - & - & - & - & - & - & - & - & + \\
\hline 82 & Pentapharsodinium sp. & - & - & + & - & - & - & - & - & - & + & - \\
\hline
\end{tabular}


Tab. 1. - continued

\begin{tabular}{|c|c|c|c|c|c|c|c|c|c|c|c|}
\hline & \multicolumn{11}{|c|}{ Stations } \\
\hline & 1 & 2 & 3 & 4 & 5 & 6 & 7 & 8 & 9 & 10 & 11 \\
\hline 83 Peridiniella sp. & + & + & + & + & - & - & - & - & - & - & - \\
\hline 84 Periperidinium $\mathrm{sp}$. & - & - & + & - & + & + & - & - & - & + & - \\
\hline 85 Podolampas palmipes Stein & - & - & - & - & - & - & - & - & - & + & - \\
\hline 86 Polykrikos schwarzii Bütschli & - & + & - & - & + & + & + & + & + & + & + \\
\hline $\begin{array}{l}87 \text { Prorocentrum balticum (Lohmann, 1908) } \\
\text { Loeblich }\end{array}$ & - & - & - & - & - & - & - & - & - & - & + \\
\hline $\begin{array}{l}88 \text { Prorocentrum compressum (Bailey) Abé ex } \\
\text { Dodge }\end{array}$ & - & - & - & - & - & - & - & - & - & - & + \\
\hline 89 Prorocentrum lima (Ehrenberg) Dodge & + & - & - & - & - & - & - & - & - & + & + \\
\hline 90 Prorocentrum mexicanum Tafall & + & + & + & + & + & + & + & + & - & + & + \\
\hline 91 Prorocentrum micans Ehrenberg & + & + & + & + & + & + & + & + & + & + & + \\
\hline 92 Prorocentrum minimum (Pavillard) Schiller & + & + & - & + & + & + & - & + & + & + & - \\
\hline 93 Prorocentrum ruetzlerianum Faust & + & - & - & - & - & - & - & - & - & - & - \\
\hline 94 Prorocentrum sigmoide Bohm & + & + & - & + & + & + & + & - & + & + & - \\
\hline 95 Prorocentrum sp. & - & - & - & - & - & - & - & - & + & - & + \\
\hline 96 Prorocentrum triestinum Schiller & + & + & + & + & + & + & + & + & - & + & + \\
\hline $\begin{array}{l}97 \text { Protoceratium reticulatum (Claparède et } \\
\text { Lachmann) Butschli }\end{array}$ & - & - & - & - & - & - & - & + & - & - & + \\
\hline 98 Protoceratium sp. & - & - & - & - & - & - & - & - & - & + & + \\
\hline 99 Protoperidinium acanthophorum (Balech) Balech & + & - & - & - & - & - & - & - & - & - & - \\
\hline 100 Protoperidinium bipes (Paulsen) Balech & + & + & + & + & + & + & + & + & + & + & + \\
\hline 101 Protoperidinium bispinum (Schiller)Balech & + & + & + & + & + & + & + & + & + & + & + \\
\hline 102 Protoperidinium brevipes (Paulsen) Balech & + & + & - & - & + & + & - & - & + & - & + \\
\hline 103 Protoperidinium capurroi (Balech) Balech & + & - & + & - & - & - & - & - & - & - & - \\
\hline 104 Protoperidinium cf. avellana (Meunier) Balech & + & - & - & - & - & - & - & - & - & - & - \\
\hline $\begin{array}{l}105 \text { Protoperidinium cf. capurroi subpellucidum } \\
\text { (E. Balech) E. Balech }\end{array}$ & - & - & - & - & - & - & - & - & - & - & + \\
\hline 106 Protoperidinium cf. nanum (Balech) Balech & - & - & - & - & - & - & - & - & - & - & + \\
\hline $\begin{array}{l}107 \text { Protoperidinium } c f \text {. obtusum (Karsten) Parke et } \\
\text { Dodge }\end{array}$ & - & - & - & - & - & - & - & - & - & + & - \\
\hline 108 Protoperidinium claudicans (Paulsen) Balech & - & - & - & - & - & - & - & - & - & + & + \\
\hline 109 Protoperidinium conicoides (Paulsen) Balech & + & + & - & - & - & - & + & - & + & + & + \\
\hline 110 Protoperidinium conicum (Gran) Balech & - & + & - & + & - & - & - & + & + & + & + \\
\hline $\begin{array}{l}111 \text { Protoperidinium conicum (Gran) Balech avr. } \\
\text { conicum in Balech }\end{array}$ & - & - & + & + & - & - & - & - & - & - & - \\
\hline $\begin{array}{l}112 \text { Protoperidinium conicum (Gran) Balech var. } \\
\text { concavum Matzenauer }\end{array}$ & - & - & - & - & - & - & - & - & - & - & + \\
\hline 113 Protoperidinium cruciferum (Balech) Balech & - & - & - & - & - & - & - & - & + & - & - \\
\hline 114 Protoperidinium curtipes (Jörgensen) Balech & - & - & - & - & - & - & - & + & + & - & . \\
\hline
\end{tabular}


Tab. 1. - continued

\begin{tabular}{|c|c|c|c|c|c|c|c|c|c|c|c|c|}
\hline & & \multicolumn{11}{|c|}{ Stations } \\
\hline & & 1 & 2 & 3 & 4 & 5 & 6 & 7 & 8 & 9 & 10 & 11 \\
\hline 115 & $\begin{array}{l}\text { Protoperidinium decipiens (Jörgensen) Parke et } \\
\text { Dodge (Jörgensen) Parke et Dodge }\end{array}$ & - & - & + & - & - & - & - & - & - & - & - \\
\hline 116 & Protoperidinium depressum (Bailey) Balech & - & + & - & - & - & - & - & - & - & - & + \\
\hline 117 & Protoperidinium diabolus (Karsten) Balech & - & + & + & + & + & + & + & + & + & + & + \\
\hline 118 & Protoperidinium divaricatum (Meunier) Balech & - & - & - & - & - & - & + & - & + & - & - \\
\hline 119 & $\begin{array}{l}\text { Protoperidinium divergens (Ehrenberg) Balech } \\
\text { (Ehrenberg) Balech }\end{array}$ & + & + & - & - & - & + & + & + & + & + & + \\
\hline 120 & Protoperidinium excentricum (Paulsen) Balech & - & - & - & - & - & - & - & - & - & - & + \\
\hline 121 & Protoperidinium fartum Balech & - & - & - & - & - & - & - & - & - & - & + \\
\hline 122 & Protoperidinium gibbosum (Mat & - & - & - & - & - & + & - & - & - & - & - \\
\hline 123 & $\begin{array}{l}\text { Protoperidinium granii (Ostenfield) Balech } \\
\text { (Ostenfield) Balech }\end{array}$ & - & - & - & - & - & + & - & - & - & - & - \\
\hline 124 & Protoperidinium hirobis Abè & + & + & + & + & + & + & - & + & + & + & + \\
\hline 125 & Protoperidinium hirobis Abè & - & - & - & - & - & - & - & - & - & + & - \\
\hline 126 & Protoperidinium incognitum (B & - & - & - & - & - & - & - & + & - & - & - \\
\hline 127 & Protoperidinium latispinum (Mangin) Balech & - & - & - & - & - & - & - & - & - & - & + \\
\hline 128 & Protoperidinium leonis (Pavillard) Balech & - & - & - & - & - & - & + & + & + & - & + \\
\hline 129 & Protoperidinium mastophorum (Balech) Ba & - & - & + & + & - & - & - & + & - & + & + \\
\hline 130 & Protoperidinium metananum (Balec) & - & - & + & + & - & - & - & + & - & - & - \\
\hline 131 & Protoperidinium minutun & - & - & - & - & + & - & - & - & - & - & + \\
\hline 132 & Protoperidinium mite $\left(\mathrm{P}_{c}\right.$ & - & + & + & - & + & - & - & + & - & + & + \\
\hline 133 & Protoperidinium nanum & - & - & + & - & - & - & - & - & - & - & - \\
\hline 134 & Protope & - & - & - & - & - & - & - & + & - & + & - \\
\hline 13 & $\begin{array}{l}\text { Protop } \\
\text { Parke }\end{array}$ & + & + & + & + & + & + & + & + & + & + & + \\
\hline 136 & $\begin{array}{l}\text { Protoperidinium obtusum (Karsten) Parke et } \\
\text { Dodge }\end{array}$ & - & - & - & - & - & - & - & + & + & - & - \\
\hline 137 & $\begin{array}{l}\text { Protoperidinium ovatum subsp. asy } \\
\text { Pouchet }\end{array}$ & + & + & + & - & - & - & - & - & + & - & - \\
\hline 138 & Protoperidinium oviforme (Dangeard) Balech & - & + & - & - & - & - & - & - & - & - & + \\
\hline 139 & Protoperidinium ovum (Schiller) Balech & - & - & - & - & - & - & - & + & - & - & + \\
\hline 140 & Protoperidinium pallidum (Ostenfeld) Balech & - & - & + & - & - & - & - & - & - & - & - \\
\hline & $\begin{array}{l}\text { Protoperidinium parapyriforme (Hermosilla) } \\
\text { Balech }\end{array}$ & - & - & - & + & + & - & - & - & - & + & + \\
\hline 142 & Protoperidinium parcum (Balech) Balech & 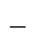 & - & - & - & - & - & - & - & + & - & - \\
\hline 143 & Protoperidinium parviventer Balech & - & - & - & - & - & - & - & - & + & - & - \\
\hline 144 & Protoperidinium paulseni Pavillard & - & - & - & - & - & - & - & - & + & - & - \\
\hline 145 & Protoperidinium punctulatum (Paulsen) Bale & $t$ & + & + & + & + & + & + & + & + & + & + \\
\hline
\end{tabular}


Tab. 1. - continued

\begin{tabular}{|c|c|c|c|c|c|c|c|c|c|c|c|}
\hline & \multicolumn{11}{|c|}{ Stations } \\
\hline & 1 & 2 & 3 & 4 & 5 & 6 & 7 & 8 & 9 & 10 & 11 \\
\hline $\begin{array}{l}146 \text { Protoperidinium pyriforme var. pyriforme } \\
\text { (Paulsen) Balech }\end{array}$ & - & - & + & - & + & - & - & - & - & - & + \\
\hline 147 Protoperidinium quarnerense (Schröder) Balech & - & - & - & - & - & - & - & - & - & - & + \\
\hline 148 Protoperidinium quinquecorne (Abé) Balech & - & - & - & + & - & - & - & - & - & - & - \\
\hline 149 Protoperidinium simulum (Paulsen) Balech & - & - & - & - & - & - & - & + & - & - & - \\
\hline 150 Protoperidinium sp. & + & + & + & - & - & - & - & + & - & + & + \\
\hline 151 Protoperidinium sphaeroideum (Mangin) Balech & - & - & - & - & - & - & - & - & + & - & + \\
\hline 152 Protoperidinium spinulosum Schiller & - & - & + & + & + & + & + & - & + & - & + \\
\hline 153 Protoperidinium steidingerae Balech & - & - & - & - & - & - & - & - & - & - & - \\
\hline 154 Protoperidinium steinii (Jørgensen) Balech & + & - & - & - & - & - & - & - & - & + & + \\
\hline 155 Protoperidinium subcrassipes E. Balech & - & - & - & - & - & - & - & - & - & - & + \\
\hline $\begin{array}{l}156 \text { Protoperidinium subpyriforme (Dangeard) } \\
\text { Balech }\end{array}$ & - & - & - & - & - & - & + & - & - & - & - \\
\hline 157 Protoperidinium subsphaericum (Broch) Balech & + & - & - & - & - & - & - & - & - & - & - \\
\hline 158 Protoperidinium thorianum (Paulsen) Balech & + & - & - & + & - & - & - & - & - & - & - \\
\hline 159 Protoperidinium ventricum (Abé) Balech & - & - & - & + & - & - & - & - & - & - & - \\
\hline 160 Protoperidinium vulgare Balech & - & - & + & - & - & - & - & - & - & - & - \\
\hline $\begin{array}{l}161 \text { Pyrocystis noctiluca Murray ex Haeckel Murray } \\
\text { ex Haeckel }\end{array}$ & - & - & - & + & - & - & - & - & - & + & - \\
\hline 162 Pyrophacus horologicum Stein & + & + & + & + & + & + & + & + & + & + & + \\
\hline 163 Pyrophacus sp. & - & - & - & - & - & - & - & - & + & - & - \\
\hline $\begin{array}{l}164 \text { Pyrophacus steinii (Schiller) } \\
\text { Wall et Dale }\end{array}$ & - & - & - & - & - & - & - & - & - & - & + \\
\hline 165 Scrippsiella precaria Montressor et Zingone & - & - & - & - & - & - & - & + & - & - & - \\
\hline $\begin{array}{l}166 \text { Scrippsiella spinifera } \mathrm{G} \text {. Honsell et } \\
\text { M. Cabrini }\end{array}$ & - & + & + & + & + & + & - & + & + & + & + \\
\hline 167 Scrippsiella sweeneyae Balech ex Loeblich III & + & - & + & + & - & + & - & + & + & + & + \\
\hline 168 Scrippsiella trochoidea (Stein) Loeblich III & + & + & + & + & + & + & + & + & + & + & + \\
\hline 169 Spirilax jollifei (Murray et Whitting) Kofoid & + & + & + & - & - & - & - & + & - & - & \\
\hline
\end{tabular}

tium furca, Gonyaulax souseae, Alexandrium minutum, Scrippsiella trochoidea, Prorocentrum triestinum and Achradina pulchra. A maximum of 201 taxa were observed at station 11, the minimum of 72 species at station 7 (Fig. 5).

\begin{abstract}
Abundance
The maximum phytoplankton abundance was found in August 2008 (Fig. 6), due to the bloom of Nitzschia longissima $\left(1.7 \times 10^{7}\right.$ cells $\mathrm{L}^{-1}$ at station 1 , located in the N-W Beninsar area, and the bloom of Skeletonema $\left(7.4 \times 10^{6}\right.$ cells $\left.\mathrm{L}^{-1}\right)$ observed at station 4 . The minimum abundance was recorded in November 2007.
\end{abstract}




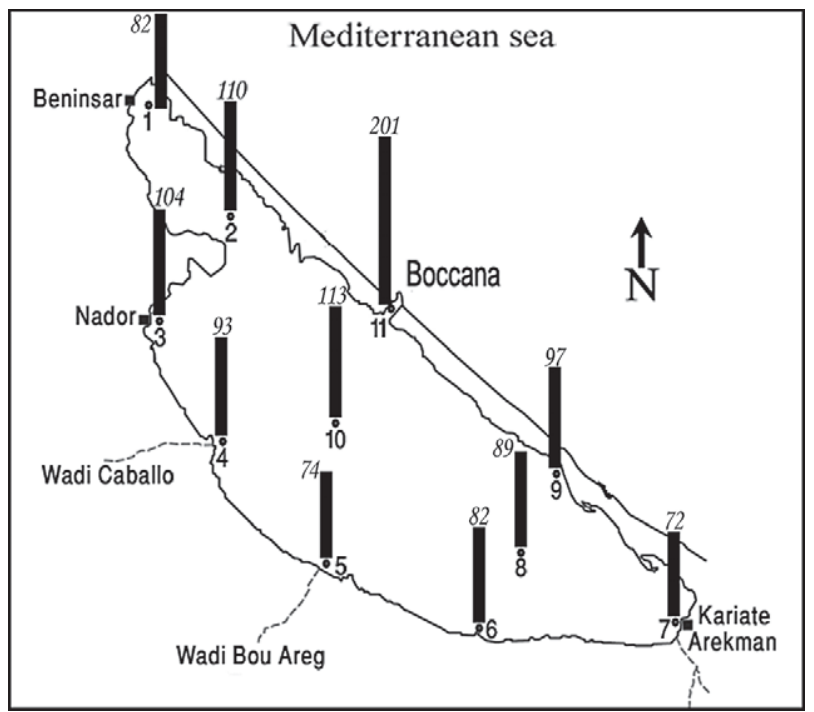

Fig. 5. Spatial distribution of the phytoplankton taxa numbers.

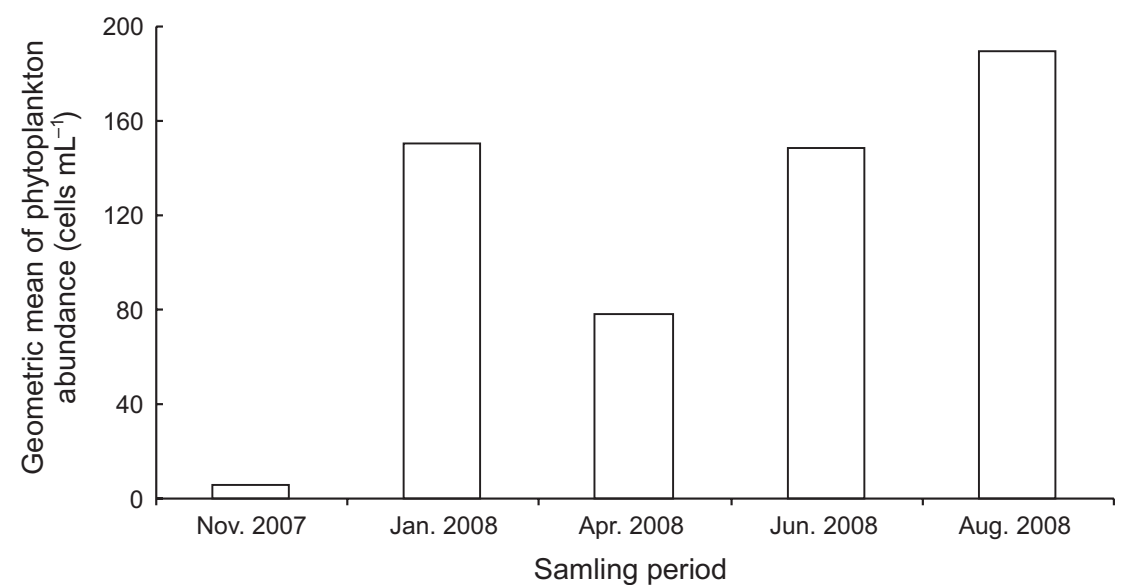

Fig. 6. Temporal variation of the geometric mean of phytoplanktonic abundance of the 11 stations.

The abundances in January and June differ due to the bloom of Chaetoceros observed in January at station 10 , with abundance exceeding $2 \times 10^{6}$ cells $\mathrm{L}^{-1}$.

November 2007 and January 2008: The community was dominated by the diatom Chaetoceros which contributed in $96.14 \%$ and $99.06 \%$ respectively in November and January.

In November, the maximum phytoplankton abundance was recorded at Station 2, with $4.4 \times 10^{4}$ cells $\mathrm{L}^{-1}$ (Fig. 7). The dominance of Chaetoceros was observed all over of the lagoon with the exception of stations 5 and 6 where Neoceratium furca dominated. 

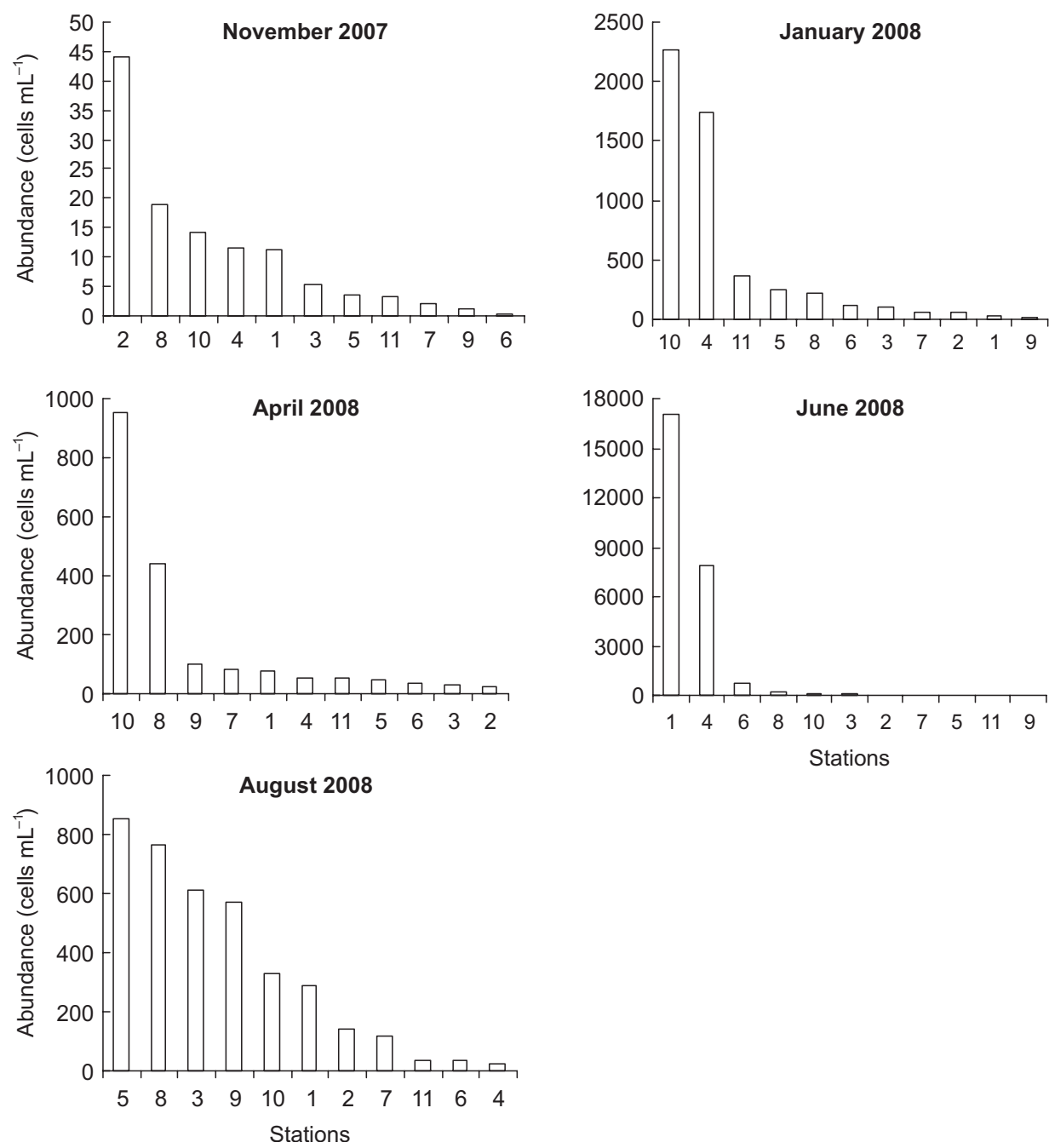

Fig. 7. Spatial variation of phytoplankton abundance during the investigated period.

In January 2008, beside the dominance of Chaetoceros, dinoflagellates such as Gonyaulax souseae, Alexandrium minutum, Scrippsiella trochoidea and Prorocentrum triestinum co-dominated at stations 1 and 2.

April 2008: High abundances were recorded at the station 10, with about $9.5 \times 10^{5}$ cells $\mathrm{L}^{-1}$ of Pseudo-nitzschia, which contributed $72.19 \%$ of the community abundance.

Jun 2008: The maximum abundance was recorded at the station 1 , with $1.7 \times 10^{7}$ cells $\mathrm{L}^{-1}$ which was mostly contributed by Nitzschia longissima $(99.9 \%$ of the community abundance).

August 2008: The maximum abundance was recorded at station 5, with $8.5 \times 10^{5}$ cells $\mathrm{L}^{-1}$ of unidentified rounded cells, 17 to $20 \mu \mathrm{m}$ in diameter (dinoflagellates or rhaphidophycean flagellatres), contributing $95.7 \%$ of the community abundance. We also found abundant 
dinoflagellate Achradina pulchra $\left(5.9 \times 10^{5}\right.$ cells $\left.\mathrm{L}^{-1}\right)$ at station 8 , and Nitzschia longissima $\left(4 \times 10^{5}\right.$ cells $\left.\mathrm{L}^{-1}\right)$ at station 3 .

The dominant species (with abundance greater than $10^{4}$ cells $\mathrm{L}^{-1}$ ) for each sampling cruise are summarized in table 2. Generally, diatoms dominated the phytoplankton community, with the occasional dominance of unidentified flagellates and dinoflagellates in August 2008.

Tab. 2. Seasonality of dominant and accompanying species in the Nador Lagoon.

\begin{tabular}{lll}
\hline sampling campaign & Dominants species & Accompanying species (Density $>10^{4}$ ) \\
\hline November 2007 & Chaetoceros spp. & Neoceratium furca (Ehrenberg) F. Gómez, D. \\
& & Moreira et P. López-García, Pseudo-nitzschia spp. \\
January 2008 & Chaetoceros spp. & Prorocentrum triestinum Schiller \\
& Small dinoflagellates $\left(^{*}\right)$ \\
& Skeletonema sp. \\
& Pseudo-nitzschia spp. \\
& Divers Bacillariophyceae pennates \\
& Nitzschia longissima (Brébisson) Ralfs \\
& & Leptocylindrus minimus Gran \\
April 2008 & Thalassionema nitzschioides (Grunow) \\
& Mereschkowsky \\
& Nitzscha longissima, (Brébisson) Ralfs \\
& Small dinoflagellates $(*)$ \\
& Chaetoceros spp. \\
& Eutreptia sp. \\
& Prorocentrum triestinum Schiller \\
\hline
\end{tabular}

(*): Alexandrium minutum, Gonyaulax verrior, G. sousea, G. dicantha Scrippsiella spp, Protoperidinium bipes, $P$. quinquecorne, P. hirobis, and others similar forms.

\section{Discussion}

In the present study, diatoms were generally dominant. The maximum number of taxa was observed at station 11, situated in Boccana channel, with a greater exchange of water between the lagoon and the open sea. The minimum number of taxa was found at station 7 located in the confined Kariat Arekman area as found in the period 1982-1993 (LEFEBVRE et al 1996). The difference in diversity between these two areas shows that more than half of the phytoplankton population comes from the open sea. The highly eutrophic conditions in the lagoon cannot maintain populations. In addition, GILABERT (2001a) explains the low diversity in the lagoon by strong physical perturbations. Anyway, more than 114 species are adapted to the lagoon's ecological conditions. About 44 phytoplankton species are common in most sampling stations (those found in at least 6 sampling stations).

The number of diatoms and dinoflagellates identified in different parts of the Mediterranean Sea mostly varies between 107 and 183 of diatoms, and 107 to 205 dinoflagellates (VILIČIĆ et al. 2002). In the Nador lagoon, we found 133 diatoms and 169 dinoflagellates. 
In the Nador lagoon we recorded a high abundance of Nitzschia longissima $\left(1.7 \times 10^{7}\right.$ cells $\mathrm{L}^{-1}$ ) in June at station 1 . This station is situated in the confined area in the north-west part of the Mar Chica that receives wastewater from Beninsar City. An increasing abundance of phytoplankton, especially diatoms to $5 \times 10^{6}$ cells $\mathrm{L}^{-1}$, usually reveals an area of anthropogenic influence (VILIČ́ć 1989).

Nador lagoon is slightly eutrophicated in October, with an tendency to increase with the approach of summer, due to the increasing temperature, light intensity and input of nutrients into the lagoon (fig. 4, 7). The eutrophication of the lagoon is manifested by the reduction of the water clarity, which is the direct result of the phytoplankton blooms, corresponding to the diatoms Chaetoceros, Pseudo-nitzschia, Nitzschia longissima etc. In summer, chlorophyll concentrations range from below $5 \mu \mathrm{g} \mathrm{L}^{-1}$ to $20 \mu \mathrm{g} \mathrm{L}^{-1}$, and oxygen decrease to anoxic conditions at stations 1 and 4. Strong eutrophic conditions were observed in the last decade. The blooms cause a green, brown or yellowish-brown discoloration of water. The bloom degradation results in increasing oxygen demand and degradation of water quality. Seasonal variations of primary production are the result of external physical chemical environmental variables and the shallowness of the lagoon (GILABERT 2001a, b).

During several years, we have observed that summer phytoplankton blooms are accompanied by appearance of abundant jellyfish Rhyzostoma pulmo. Occurrences were reported in the Mar Menor, where these organisms may play an important role in controlling eutrophication by feeding on diatoms and zooplankton (PÉREZ-RUZAFA et al. (2002).

The qualitative and quantitative results lead us to conclude that, in general, the Nador lagoon seem to be highly affected by eutrophication. A new channel (300 m wide) is going to be opened to increase the lagoon water exchange with the open sea, with an expected reduction of the residence time of water in the lagoon. This action is expected to have a great impact on the water quality by reducing the present eutrophication level, which will be accompanied by the modification of current phytoplankton population structure.

\section{Acknowledgements}

The authors thank Saida MotiA and Najib El OUAMARI for their comments on the first version, and Professor Amany Ismael for the review of the last version of the paper; Siham EL MADANI for language verification.

\section{References}

BALECH, E., 1988: Los dinoflagelados del Atlántico Sudoccidental. Publicaciones especiales del Instituto Español de Oceanografía 1, 1-310.

Balech, E., Akselman, R., Benavides, H. R., Negri, R. M., 1984: Suplemento a los dinoflagelados del Atlántico Sudoccidental. Revista de Investigación y Desarrollo de Pesquero 4, 5-20.

Benbrahim, S., 2009: Etude de l'eutrophisation de la lagune de Nador. Rapport Plan D’action Pour La Méditerranée / Programme MED POL-IV, Casablanca, 1-63.

Blound, M. K., 2005. Etude géochimique de la lagune de Nador (Maroc oriental): Impacts des facteurs anthropiques. PhD Thesis, Université de Strasbourg, Strasbourg. 
Delgado, M., Fortuño, J. M., 1991: Atlas de fitoplancton del Mar Mediterráneo. Scientia Marina 55 (Supplement1), 1-133.

DodGe, D. J., 1982: Marine dinoflagellates of the British Isles. Her Majesty's Stationery Office, University of London, London.

El Madani, F., Rharbi, N., RAmdani, M., Berraho, A., 2001: Composition and structure of phytoplanktonic communities in the Lagoon of Nador - Morocco. Rapport Commission Internationale pour l'Expoloration Scientifique de la Mer Mediterranee 36, 380.

Faust, M. A., Larsen, J., Moestrup, Ø., 1999: Potentially toxic phytoplankton: Genus Prorocentrum (Dinophyceae). International Council for the Exploration of the Sea Identification Leaflets Plankton. LEAFLET No. 184, 1-22.

GILABERT, J., 2001a: Seasonal plankton dynamics in a Mediterranean hypersaline coastal lagoon: The Mar Menor. Journal of Plankton Research 23, 207-217.

GILABERT, J., 2001b: Short-term variability of the planktonic size structure in Mediterranean coastal lagoon. Journal of Plankton Research 23, 219-226.

HallegraefF, G. M., 1991: Aquaculturist's guide to harmful Australian microalgae. Fishing Industry Training Board of Tasmania/CSIRO Division of Fisheries, Hobart.

Hallegraeff, G. M., Bolch, J. C., Blackburn, S. I., OshimA, Y., 1991: Species of the toxigenic dinoflagellate genus Alexandrium in Southeastern Australian Waters. Botanica Marina 34, 575-587.

Hansen, G, Turquet, J., Quod, J., P., Ten-Hage, L., Lugomela, C., Kyewalyanga, M., Hurbungs, M., Wawiye, P., Ogongo, B., Tunje, S., Rakotoarinjanahary, H., 2001: Potentially harmful microalgae of the western Indian Ocean - a guide based on a preliminary survey. I.O.C. Manuals and Guides 41, UNESCO.

HiLmi, K., 2005: Modélisation numérique de la circulation de deux milieux paraliques du littoral marocain: La lagune de Oualidia (Atlantique) et la lagune de Nador (Méditerranée). PhD. Thesis, Faculté des Sciences Ben M'Sik, Université Hassan II - Mohammedia, Casablanca.

KASHIMA, K., 2002: The finding of some diatoms species, which are known as brackish species from hyper-saline inland lakes in Turkey. Laguna 9, 47-53.

KoenInG, M. L., LIRA, C. G., 2004: The genus Ceratium Schrank (Dinophyta) in the continental shelf and oceanic waters of the State of Pernambuco, Brazil (O gênero Ceratium Schrank (Dinophyta) na plataforma continental e agues oceânicas do Estado de Pernambuco, Brasil). Acta Botanica Brasilica 19, 391-397.

Lakhdar, I. J., Orbi, A., Hilmi, K., Zidane, F., Moncef, M., 2005: Aquatic ecosystem modelling approach: Temperature and water quality models applied to Oualidia and Nador lagoons. Environmental Technology 26, 717-720.

Larsen, J., Moestrup, Ø., 1989: Guide to toxic and potentially toxic marine algae. The Fish Inspection Service, Ministry of Fisheries, Copenhagen.

Lefebvre, A., Guelorget, O., Perthuisot, J. P., DAfir, J. E., 1996: Evolution biogéologique de la lagune de Nador (Maroc) au cours de la période 1982-1993. Oceanologica Acta 20, 371-385.

MATSUOKA, K., FuKUYO, Y., 2000: Technical guide for modern dinoflagellate cyst study. WESTPAC-HAB/ WESTPAC/IOC, Tokyo. 
Mahjoubi, R., Kamel, S., El Moumni, B., Noack, Y., Parron, C., 2003: Nature, origine et répartition de la phase argileuse de la lagune de Nador (Maroc nord oriental). Geologica Belgica 6, 31-42.

NeZan, E., Piclet, G., 1996: Guide pratique à l'usage des analystes du phytoplankton. IFREMER - Direction de l'Environnement et de l'Aménagement Littoral, Concarneau.

NEZAN E., 1996: Surveillance du phytoplancton marin: manuel illustré adapté à la formation des analystes. IFREMER - Direction de l'Environnement et de l'Aménagement Littoral, Concarneau.

PAUlmier, G., 1992: Catalogue illustré des microphytes planctoniques et benthiques des côtes normandes. Rapport IFREMER - RIDRV-92.007-RH/Le Robert, Issy les Moulineaux.

PAUlmier, G., 1994: Les dinophycées pélagiques et benthiques du Golf de Gascogne sud de la Bretagne à Arcachon. Annales de la Société des Sciences Naturelles de la Charente-Maritime 8, 290-344.

Pérez-Ruzafa, A., Gilabert, J., Gutiérrez, J.M., Fernández, A.I., Marcos, C., Sabah, S., 2002: Evidence of a planktonic food web response to changes in nutrient input dynamics in the Mar Menor costal lagoon, Spain. Hydrobiologia 475/476, 359-369.

RICARD, M., 1987: Atlas du phytoplancton marin, 2, Diatomophycées. Centre National de la Recherche Scientifique, Paris.

Sar, E. A., Ferrario, M. E., Reguera, B., 2002: Floraciones algales nocivas en el Cono Sur Americano. Instituto Español de Oceanografia, Madrid.

Tomas, C. R., 1997: Identifying marine phytoplankton. Academic Press, San Diego.

VILIČIĆ, D., 1989: Phytoplankton population density and volume as indicators of eutrophication in the eastern part of the Adriatic Sea. Hydrobiologia 174, 117-132.

ViLičić, D., Marasović, I., Mioković, D., 2002: Cheklist of phytoplankton in the eastern Adriatic Sea. Acta Botanica Croatica 61, 57-91. 